\title{
Relative changes with contraction in the central excitability state of the tibialis anterior and calf muscles
}

\author{
MORRIS A FISHER
}

From the Laboratory of EMG and Clinical Neurophysiology, Department of Neurological Sciences, RushPresbyterian-St. Luke's Medical Center, Chicago

SUMMARY F responses were recorded from the surface of the tibialis muscle and medial aspect of the soleus muscle in 14 normal subjects. The persistence (that is the fraction of measurable $F$ responses found with a series of supramaximal stimuli) and average $F$ amplitudes (measured peak-topeak and based on at least five $F$ responses) were determined both at rest and with isometric contraction with the ankle maintained at $90^{\circ}$. Although the persistence at rest was significantly less in the tibialis anterior soleus than the $(\mathrm{p}<0.001)$, no significant difference was found with the muscles contracted. This was associated with a significant increase in both average $F$ amplitudes and average $\mathrm{F}$ amplitude/direct motor response ratios in the tibialis anterior in comparison to the soleus. In four of the subjects, studies were also performed when the $\mathrm{H}$ reflex in the soleus muscle was eliminated by thigh compression. Comparable changes in both $\mathrm{F}$ response persistence and average $\mathrm{F}$ amplitude were found with and without an $\mathrm{H}$ reflex. These data indicate that, in contrast to the situation at rest, with isometric contraction the "central excitatory state" of the tibialis anterior is at least as great as in its antagonist antigravity muscles and that this is not due simply to increased large fiber reflex input associated with agonist contraction.

Low ampliture responses of somewhat variable amptitude and latency are found in a wide distribution after supramaximal stimulation of motor nerves. These $F$ responses reflect recurrent motoneuron discharge and are intriguing in that there is evidence in both animals ${ }^{1}$ and man $^{2}$ that these responses can measure excitability states in motoneuron pools.

Previous work has shown that the prominence of the $F$ response is not uniform but varies in a distribution consistent with the physiological role of a muscle. ${ }^{3}$ In particular, $F$ responses are found more readily and are generally of larger amplitude in antigravity muscles in comparison to their antagonists. This relation can be altered in patients with abnormal motor states and modified by cerebellar stimulation. ${ }^{4}$

It is the purpose of this paper to amplify on these observations by demonstrating that facilita-

\footnotetext{
Address for reprint requests: Dr MA Fisher, Department of Neuro'ogical Sciences, Rush-Presbyterian-St. Luke's Medical Center, $1753 \mathrm{~W}$ Congress Parkway, Chicago, Illinois 60612, USA.

Accepted 10 October 1979
}

tion of $\mathrm{F}$ responses by agonist contraction is also not uniform and that these differences can presumptively be related to the physiological organisation of a particular muscle.

\section{Methods}

$F$ responses were obtained in 14 control subjects without any neurological abnormality. The ages of these subjects ranged from 26 to 45 years with a mean age of 35 . Responses were recorded from the medial aspect of the soleus (S) stimulating the posterior tibial nerve in the popliteal fossa and from the tibialis anterior (TA) stimulating the peroneal nerve at the fibula head. Muscle belly recordings were used with the recording electrode (TECA 6030) placed one-half the distance between the mid-popliteal crease and the medical malleolus for the $S$ and one-third the distance between the tibial tubercle and the dorsal ankle crease for the TA. The indifferent electrode was placed $50 \mathrm{~mm}$ distally. Stimuli (TECA NS6) were given at a rate of $0.5 \mathrm{~Hz}$ with the stimulus duration $0 \cdot 2 \mathrm{~ms}$ and the voltage at a level twenty- 
five per cent supramaximal for the direct motor (M) response. During the studies, the subjects were supine with the limb restrained in a short leg brace such that the ankle was maintained at an angle of $90^{\circ}$.

Recordings were made both at rest and during maximum isometric contraction of the calf and TA muscles. When recording from a muscle at rest a series of stimuli were used until at least 10 clearly measurable $F$ responses (ie greater than $50 \mu \mathrm{V}$ in amplitude) were obtained and/or a maximum of 20 stimuli were given. Similar criteria were employed for recordings during muscle contraction except that a maximum of 20 stimuli were given in which there were clearly $10 \mathrm{~F}$ responses and/or $\mathrm{F}$ responses could reasonably be considered absent; that is, the $F$ responses either occurred or could have expected to be seen during the relative absence of background activity associated with a silent period. Throughout, simultaneous recordings were made from the $S$ and TA in order to analyse the effect of "spread" of electrical activity.

In four subjects, similar studies were performed at a time when $\mathrm{H}$ reflexes were no longer obtained from the soleus either at rest or with facilitation by calf contraction. $\mathrm{H}$ reflexes were inhibited by thigh compression with a blood pressure cuff elevated above systolic pressure. After approximately 20 to 25 minutes, a period was present in which $\mathbf{H}$ reflexes were absent while $M$ and associated $F$ responses were relatively unihibited.

All data were recorded on photosensitive paper. The following data were analysed at both rest and contraction with and without thigh compression: maximum $\mathbf{M}$ amplitudes measured peakto-peak; $F$ persistence (Fp), ie the number of measurable $F$ responses divided by the number of supramaximal stimuli; and, average $F$ amplitudes (avF) as well as average $F$ amplitude divided by maximum $\mathbf{M}$ amplitude (avF/M) ratios if five or more measurable $F$ responses were obtained, ie if the Fp were $\mathbf{0 . 2 5}$ or greater.

The studies are, of course, routine, electrophysiological procedures and entirely harmless. The subjects were all personally acquainted with the procedures and informed consent obtained.

Statistical analysis of comparison of means was performed by t-tests.

\section{Results}

The main results of this study are shown in figs 1 an 12 as well as the table. There is clearly a

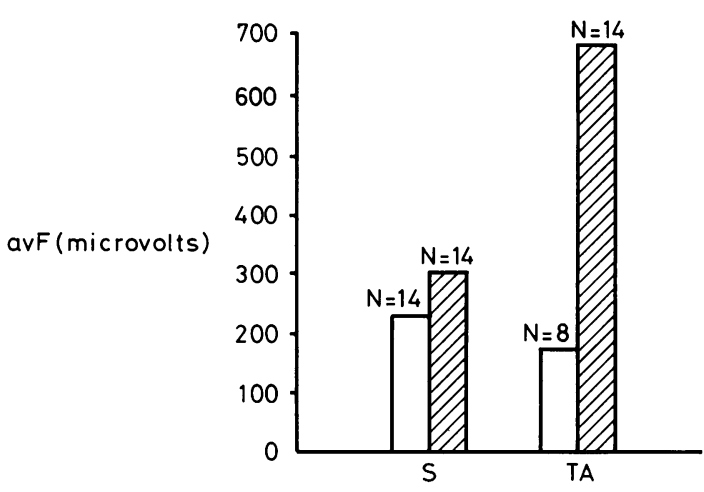

Fig 1 Facilitation of average $F$ amplitudes (avF) recording from the soleus (s) and tibialis anterior (TA) muscles. Clear bars at rest; crossed bars with contraction. $N=$ number of observations.

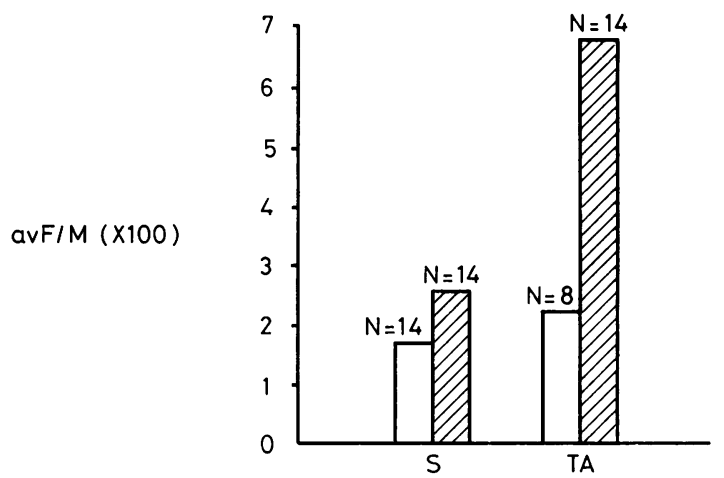

Fig 2 Same as in fig 1 except comparing average $F$ amplitude divided by direct motor response $(a v F / M)$ ratios.

relatively greater increase with contraction of the responses in the TA in comparison to the $S$. Whereas the minimum increase in avF amplitudes with contraction divided by that at rest for the TA was $1 \cdot 8$, this degree of increase was found in only two of the 14 studies in S. In another three of these same 14 studies in the $\mathrm{S}$, there was a decrease in the avF amplitudes with contraction in comparison to that at rest (ratios 0.69-0.78).

At rest, $F$ response amplitudes tended to be greater in the $S$ than the TA with avF amplitude ratios for the TA/S $(\mathrm{N}=8)$ ranging from 0.36 to 0.94 (mean $=0.62$ ). The differences in Fp were more marked, with the range at rest for the S 0.83 to 1.00 while that for the TA 0.07 to $1 \cdot 00$. In six of these 14 studies in the TA, the Fp was less than 0.25 (range 0.07 to 0.20 ) so that avF amplitude values for these studies have not been included in the data (see Methods). For 
those eight subjects in which $\mathrm{Fp}$ in the $\mathrm{TA}$ at rest was greater than $0 \cdot 25, \mathrm{avF} / \mathrm{M}$ ratios were not significantly different from comparable ratios found in the $S$.

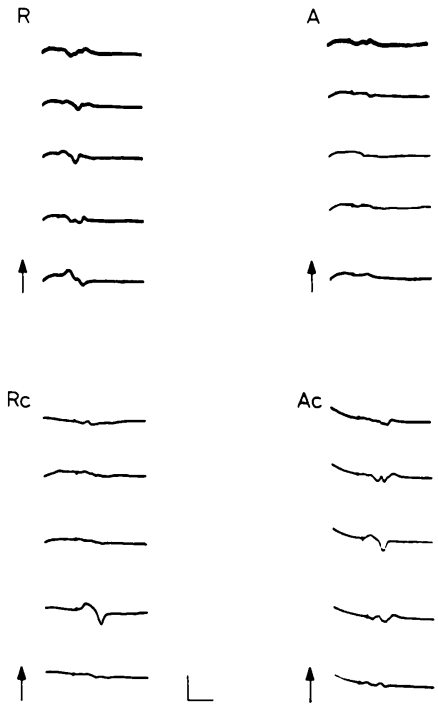

Fig 3 Representative $F$ responses recording from the $S$ muscle at rest $(R)$ and contraction $(A) . R c$ and Ac same except $F$ responses obtained when $H$ reflex inhibited by thigh compression. Note absence of differences in the responses whether $H$ reflexes present or absent except for increase in $F$ response latencies. Arrows-stimuli. Calibration-500 $\mu \mathrm{v}$ and $10 \mathrm{~ms}$
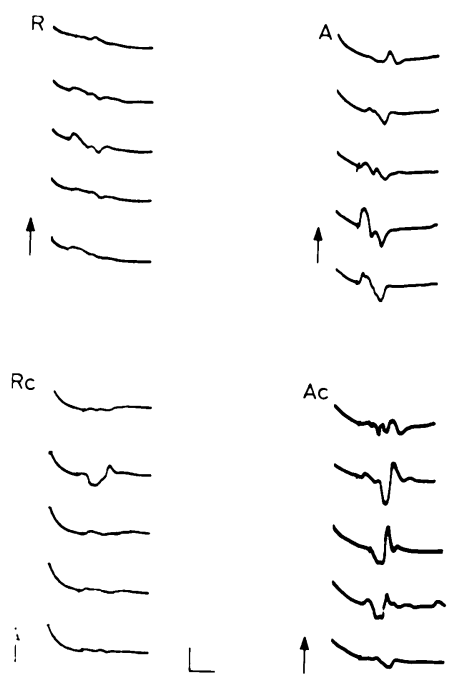

Fig 4 Same as fig 3 except recording from $T A$. Note prominent facilitation of $F$ responses with muscle contraction.
Fig 3 shows representative $F$ responses in the $\mathrm{S}$ and TA. The absence of change at a time when $\mathrm{H}$ reflexes were no longer present in the $\mathrm{S}$ as a result of thigh compression is shown. In the four subjects in which such studies were performed, data were obtained from both the $\mathrm{S}$ and TA at both rest and contraction in two subjects while in the other two such data were not obtained from the $S$ in the other. At the time of attempted recordings in these two subjects, $F$ responses were not seen in those muscles associated with a greater than fourfold decrease in the $\mathbf{M}$ responses.

Ratios of avF amplitudes in the soleus at contraction and at rest when $\mathrm{H}$ reflexes were not present were $0 \cdot 65,1 \cdot 14$, and $1 \cdot 94$ while comparable values in the same subject prior to thigh compression were $0 \cdot 69,1 \cdot 55$, and $2 \cdot 1$. Similar

Table $F$ response amplitude, persistence, and $a v F / M$ at rest and contraction*

\begin{tabular}{|c|c|c|c|c|c|}
\hline & & \multicolumn{2}{|l|}{ Rest } & \multicolumn{2}{|c|}{ Contractions } \\
\hline & & $S$ & $T A$ & \multicolumn{2}{|c|}{$\bar{S} \quad T A$} \\
\hline Average amplit & $\mathrm{e}(\mu \mathrm{v}) \mathrm{N}_{2}$ & $225 \pm 42$ & 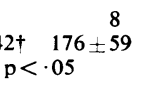 & $\begin{array}{r}14 \\
304 \pm 100 \\
p\end{array}$ & $\begin{array}{c}14 \\
679 \pm 286 \\
<0.001\end{array}$ \\
\hline $\mathrm{Fp}$ & $\begin{array}{c}\mathrm{N} \\
0 \cdot 96=0\end{array}$ & $\begin{array}{c}14 \\
0.005\end{array}$ & $\begin{array}{c}14 \\
0.51 \pm 0.34 \\
\mathrm{p}<0.001\end{array}$ & $0.97 \pm 0.7$ & $\begin{array}{c}14 \\
0.92 \pm 12 \\
\text { NS }\end{array}$ \\
\hline $\mathrm{avF} / \mathrm{M}(\mathrm{x} 100)$ & ${ }^{N}$. & $\begin{array}{c}14 \\
8 \pm 0 \cdot 5 \\
1\end{array}$ & $\begin{array}{lc} & 8 \\
54 & 2 \cdot 4 \pm 1 \cdot 4 \\
\text { NS } & \end{array}$ & $\begin{array}{c}14 \\
2 \cdot 7 \pm 1 \cdot 5 \\
p\end{array}$ & $\begin{aligned} & 14 \\
& 6 \cdot 9 \pm 2 \cdot 8 \\
&<0 \cdot 001\end{aligned}$ \\
\hline
\end{tabular}

$\mathrm{N}=$ Number of observations; $\uparrow$ mean \pm Standard Deviation

NS $=$ Not significant $(p<0.05)$

*=-For abbreviations see methods

data when recording from the TA in two of the subjects were $2 \cdot 0$ and $3 \cdot 3$ with thigh compression and 2.5 and 4.7 before. In the third subject, Fps from the TA at rest were less than 0.20 while these values with contraction were $1 \cdot 00$. This change was associated with avF/M ratios of 0.082 before and 0.070 after thigh compression. Fps were comparable in the same subjects whether $\mathrm{H}$ reflexes were present or not and $\mathrm{avF} / \mathrm{M}$ ratios were similar except in one subject. In this subject, when recording from the soleus, the data indicated approximately a twofold increase in the avF/ $M$ ratio due to a relatively large decrease in the maximum $\mathbf{M}$ amplitude in comparison to the avF amplitude.

\section{Discussion}

This study indicates that facilitation of "late" responses with supramaximal stimulation is 
relatively greater in the $T A$ than the antagonist calf muscle. This relationship remains true even when conduction in the larger afferent fibres is blocked due to thigh compression. The experimental observations therefore cannot be explained solely by reflex activation of motoneurons associated with agonist contraction. Even without these findings, reflex effects would seem unlikely as an explanation for the data. Agonist contraction consistently facilitates reflex responses obtained with submaximal stimulation, that is $\mathbf{H}$ reflexes in the calf muscles, while such responses are not normally found at rest in the TA. Even with contraction, in our experience, $\mathrm{H}$ reflexes in the TA are elicited consistently only with facilitation by double stimulation. ${ }^{3}$ Since this is true at submaximal stimulation, there is no evidence that reflex effects would be more prominent in the TA than the calf muscles with supramaximal stimulation even with agonist contraction. It seems reasonable, therefore, to assume that the experimental data reflect primarily the effect of antidromic activation of motoneurons, namely $F$ responses.

The prominence of $F$ responses is clearly less, as a rule, in the TA than the calf muscles. The most meaningful value in this regard is the Fp since differences in amplitudes could relate to differences in organisation of motor units, anatomical features of muscle fibres, and pattern of spread of electrical activity in the different muscles. Nevertheless, the observed fact is that, using the methods of this study, avF amplitudes also tend to be lower in the TA than when recording from the $S$. These differences are consistent with the hypothesis that the resting "central excitatory state" in the antigravity calf muscles is greater than its antagonist TA. When $F$ responses are found with some regularity in the $\mathrm{TA}$, avF/M ratios in this study were not found significantly different from those in the calf. This would suggest that a manifestation of the differences in excitability between the motoneuron pools is a generalised difference in the likelihood of any recurrent motoneuron activation.

The usual difference in $F$ response amplitudes when recording with surface electrodes from the TA and $S$ is relevant for another, practical reason. Given the techniques of this study, there is the possibility of spread of electrical activity from one muscle to another. $F$ response activity from the TA when stimulating supramaximally at the popliteal fossa and recording from the $S$, is rarely a problem because of the relatively low amplitude and infrequent responses from the TA.
A more serious problem is that, with supramaximal stimulation at the fibular head, one may stimulate the posterior tibial nerve submaximally resulting in a relatively large $H$ reflex in the calf muscle. "Cross talk" from this activity can obviously interfere with recordings from the TA. In two subjects, data could not be used for this study due to failure to eliminate this problem by electrode positioning.

The difference in facilitation with contraction in the TA and calf muscles can be related to differences in their physiological organisation. A relatively increased "central excitory state" in the antigravity calf muscles could be important for maintaining tonic motoneuron activity in these muscles. Given the conditions of this study, calf contraction might produce relatively little facilitation in that segment of the motoneuron pool antidromically activated, and some of the motoneurons recurrently discharged might be blocked because of rapid depolarisation and resultant failure of the impulse to pass through the still depolarised axonal initial segment. ${ }^{67}$

$F$ responses are composed of discharges in only a limited number of motor units ${ }^{8}$; only a proportion of the motor units in a particular pool can be fired antidromically; and, even for those motor units antidromic activation occurs only infrequently. ${ }^{7}$ At the same time, there is general agreement that measurement of $F$ responses $(10$ to 20) can provide a measure of conduction in the larger motor fibres. ${ }^{9}$ This could only be true if there were a selective activation with antidromic stimulation of the larger motoneurons with their associated larger axons. Which proportion of a motoneuron pool is excited by a particular facilitory manoeuver may therefore be important in determining the response observed after supramaximal stimulation.

The relatively increased facilitation in the physiological flexor TA in comparison to the physiological extensor calf muscles observed in this study may be a manifestation of a more general principle of motor system organisation. Group II afferents of whatever origin facilitate flexors and inhibit extensors, and there is evidence that the facilitory effects of long-loop reflexes ${ }^{10}$ and Renshaw cell firing ${ }^{11}$ are also more prominent in flexor muscles. Even if resting excitability is greater in certain postural muscles, it would seem reasonable, that with activity a more balanced state would be desirable. It is also reasonable to think that distortion of this balance would be a feature of at least certain disordered motor states. 


\section{References}

1 Gassel MM, Marchiafava PL, Pompeiano $O$. Modulation of the recurrent discharge of alpha motoneurons in decerebrate and spinal cats. Arch Ital Biol 1965; 103:1-24.

2 Fisher MA, Shahani BT, Young RR. Assessing segmental excitability after acute rostral lesions. Neurology (Minneap) 1978; 28:1265-71.

3 Fisher MA. Electrophysiological appraisal of relative segmental motoneurone pool excitability in flexor and extensor muscles. J Neurol Neurosurg Psychiatry 1978; 41:624-9.

4 Fisher MA, Penn RD. Evidence for changes in segmental motoneurone pools by chronic cerebellar stimulation and its clinical significance. J Neurol Neurosurg Psychiatry 1978; 41:630-5.

5 Garcia HA, Fisher MA, Gilai A. H reflex analysis of segmental reflex excitability in flexor and extensor muscles. Neurology (Minneap) 1979; 29:984-91.
6 Eccles JC. The central action of antidromic impulses in motor fibers. Pfluegers Arch 1955; 260:385-415.

7 Schiller HH, Stalberg E. F response studied with single fibre EMG in normal subjects and spastic patients. J Neurol Neurosurg Psychiatry 1978; 41:45-53.

8 Feasby TE, Brown WF. Variation of motor unit size in the human extensor digitorum brevis and thenar muscles. J Neurol Neurosurg Psychiatry 1974; 37:916-26.

9 Young RR, Shahani BT. Clinical valve and limitations of F-wave determination. Muscle Nerve 1978; 1:248-9.

10 Shimamura $M$, Akert K. Peripheral nervous relations of propriospinal and spino-bulbo-spinal reflex systems. Jpn J Physiol 1965; 15:638-47.

11 Wilson VJ, Talbot WH, Diecke FPJ. Distribution of recurrent facilitation and inhibition in cat spinal cord. J Neurophysiol 1960; 23:144-53. 